\title{
Complexities of transcatheter mitral valve replacement (TMVR) and why it is not transcatheter aortic valve replacement (TAVR)
}

\author{
Moritz C. Wyler von Ballmoos ${ }^{1,2}$, Ankur Kalra ${ }^{3}$, Michael J. Reardon ${ }^{1,2}$ \\ ${ }^{1}$ Department of Cardiovascular Surgery, Houston Methodist Hospital, Houston, Texas, USA; ${ }^{2}$ Weill Cornell Medicine, New York, NY, USA; \\ ${ }^{3}$ Division of Cardiovascular Medicine, Department of Medicine, Harrington Heart \& Vascular Institute, University Hospitals Cleveland Medical \\ Center, Case Western Reserve University School of Medicine, Cleveland, OH, USA \\ Correspondence to: Moritz C. Wyler von Ballmoos, MD, PhD, MPH, FACC, FAHA. Department of Cardiovascular Surgery, Houston Methodist \\ DeBakey Heart \& Vascular Center, Houston, TX 77030, USA. Email: mcwylervonballmoos@houstonmethodist.org.
}

\begin{abstract}
Transcatheter mitral valve replacement (TMVR) is currently being investigated as a procedural alternative to surgical mitral valve repair or replacement (SMVR). Early data from first-in-man trials with current devices suggest that TMVR is technically feasible but carries a high mortality. This is substantially different from the early success transcatheter aortic valve replacement (TAVR) has seen and is related to complexities of the mitral valve anatomy, differences in pathology that require mitral valve replacement as well as the impact that mitral valve replacement has on physiology and cardiac function, irrespective of the modality by which the mitral valve is replaced. Importantly, in the case of TAVR, a less invasive method is offered to accomplish the same as the traditional surgical intervention. On the other hand, valve replacement is not the recommended treatment option for the majority of mitral valve disease, and in fact is avoided whenever possible during surgery given the shortened life expectancy and increased morbidity with mitral valve replacement. Another distinction between TAVR and TMVR is the etiology and natural progression of the underlying disease and driving factors for intervention that are vastly different between aortic and mitral valve disease. The primary aortic disease treated has been aortic stenosis, which has several etiologic factors that cause a similar physiologic dysfunction and risk. Aortic valve replacement leads to improved survival and quality of life. The primary mitral valve disease targeted is regurgitation, which occurs as a primary valve defect and as a secondary consequence of ventricular dysfunction. Primary mitral regurgitation is treated by valve repair with excellent long-term outcomes. Secondary regurgitation has poor long-term outcomes with current commonly used repair techniques and limited data exists showing that correction of the regurgitation improves survival. Adoption of TMVR will require overcoming the anatomic challenges as well as generating data that supports improved survival and/or quality of life.
\end{abstract}

Keywords: Mitral valve; transcatheter mitral valve replacement (TMVR); transcatheter aortic valve replacement (TAVR); transcatheter therapy; device development

Submitted Aug 07, 2018. Accepted for publication Oct 04, 2018.

doi: $10.21037 /$ acs.2018.10.06

View this article at: http://dx.doi.org/10.21037/acs.2018.10.06

\section{Introduction}

Following the success and rapid adoption of transcatheter aortic valve replacement (TAVR), a procedural, catheterbased approach to mitral valve pathology and options for replacement of the mitral valve quickly became an obvious target for investigators and industry. Whilst TAVR has become commonplace for aortic stenosis in patients that present with an increased surgical risk, transcatheter mitral valve replacement (TMVR) is still very much in its infancy. To date, only about 250 TMVR cases with a dedicated mitral valve system have been performed worldwide. The only randomized trial for a transcatheter mitral valve device in the United States is currently the TMVR with 


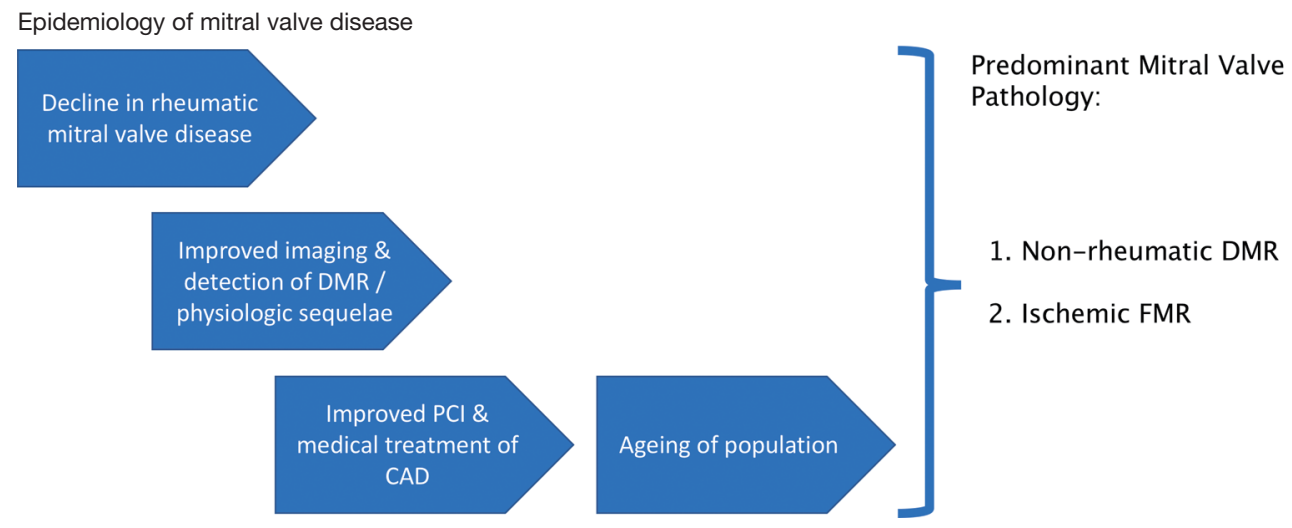

Figure 1 Epidemiology of mitral valve pathology. DMR, degenerative mitral valve regurgitation; PCI, percutaneous coronary intervention; CAD, coronary artery disease; FMR, functional mitral regurgitation.

the Medtronic Intrepid ${ }^{\mathrm{TM}}$ TMVR System in Patients with Severe Symptomatic Mitral Regurgitation (APOLLO) trial (NCT03242642), investigating the Intrepid valve. Initial results from this study are expected to be available in 2021 . In 2015 alone, the total investments made by industry in mitral valve technology exceeded 2.5 billion U.S. dollars (1). This is consistent with the much higher incidence of mitral valve pathology in the general population. It is estimated that in the United States, 1.7 percent of the adult population are living with mitral regurgitation and a small percentage with mitral stenosis (2).

\section{Different patient characteristics affecting the need for TMVR and its feasibility}

Over the last several decades, there has been a dramatic reduction in the prevalence of rheumatic heart disease in developed countries. The aging of our population combined, with the advent of advanced medical and interventional therapy for ischemic heart disease, have led ischemic functional secondary mitral regurgitation (MR) and degenerative primary MR to become the most common mitral valve pathologies $(2,3)$. Mitral regurgitation is now the most common valve pathology overall (Figure 1). With the shift from mitral stenosis to MR over time, the needs for therapeutic interventions in the mitral space have morphed away from valve replacement and towards valve repair (see also next section 'Challenges with mitral valve replacement in general').

The demographics of patients presenting with degenerative aortic valve disease and mitral valve disease are also distinctively different. A review of the Society of
Thoracic Surgeons (STS) database shows that patients undergoing surgical aortic valve replacement are on average are 70 years old, with $20 \%$ of all patients being octogenarians or older (4). Contrary to that, patients requiring mitral valve replacement are 10 years younger on an average and only $8 \%$ are 80 years old or older (5). This is a critically important context for the durability of bioprosthetic valves and the resulting need for future interventions. It is well established that bioprosthetic valves have a shortened lifespan in younger patients, arguably due to a more robust immune response, and heightened hemodynamic stress in younger patients. Both the shortened durability of valves and longer life expectancy in younger patients will contribute to a higher incidence of subsequent valve replacement. It is well known from surgical valve replacements that mitral valves have a higher rate of degeneration than aortic valves. This is likely because the mitral valve is exposed to a systolic pressure gradient and the aortic valve to a diastolic pressure gradient. Additionally, valve-in-valve procedures create a tube graft by the second valve continuously holding open the first valve. In the aortic position, the structure at risk is the coronary circulation; the likelihood of an occlusion can be predicted relatively reliably from CT scans based on the size of the Sinuses of Valsalva. In the mitral position, the 'tube graft' will interfere with the left ventricular outflow track (LVOT) and the extent of this obstruction is much more difficult to predict. Additionally, the consequences of complications, e.g., the need for a permanent pacemaker and suboptimal hemodynamic results, such as residual gradients or paravalvular leaks, are more impactful, again due to the longer life expectancy of younger patients. 
Additionally, the changes in valve morphology requiring replacement are quite different between the aortic and mitral position. TAVR has become a tremendous success for treatment of the most common aortic valve pathology, degenerative sclerotic aortic stenosis. Bicuspid aortic valves [about $10 \%$ of aortic stenosis (AS) cases in Western countries; roughly 20\% in Asian countries] and predominant aortic insufficiency (13\% of AVR cases) (6) continue to pose a challenge for good outcomes with TAVR. In case of the mitral valve, the underlying pathology that will require valve replacement comes in many more variations. The mitral valve forms a complex, functional unit with its subvalvular apparatus. The varying degree of excessive tissue or scarring of leaflets, chordal rupture, annular dilatation, leaflet and/or annular calcification create a sheer unlimited number of substrates in which a transcatheter valve will have to be deployed successfully.

Learning from the TAVR experience, implantation of an aortic transcatheter heart valve (THV) in the mitral position has been performed successfully in cases of severe mitral annular calcification (MAC) (7). Because the valves used were not specifically designed for the mitral position, the success of these cases can be attributed in part to the annular calcification, a condition that is uncommon in most patients with mitral valve disease.

\section{Challenges with mitral valve replacement in general}

The predominant presenting pathology in mitral valve disease is regurgitation, either due to primary degenerative valve disease or with ischemic functional secondary $M R$. Other than with AS, mitral valve disease is rarely associated with sudden or rapid progression to death, and the need for intervention is mostly driven by symptoms. Although MR does shorten life expectancy, this effect is more indolent, occurring over years, as opposed to months as seen in AS patients. Guideline-directed medical therapy has high 1-year survival in mitral valve patients. It is widely accepted and emphasized in guidelines that surgical repair is the preferred approach over replacement in primary MR because of superior outcomes, both in terms of morbidity and mortality (8). Today, over $60 \%$ of patients in the U.S. undergoing mitral valve surgery have a valve repair (4) and for primary degenerative mitral valve disease, the need for valve replacement in experienced centers is nearly nonexistent (9). On the other hand, the most appropriate approach to secondary MR remains controversial in the light of recent evidence that puts the durability of surgical repair in this group of patients into question $(10,11)$. Highrisk patients with functional secondary MR may therefore become the most likely first demographic in which TMVR could be successful and prove useful. The trouble with this growing group of patients is that the mitral valve is not actually the problem and any intervention on it will not treat the underlying ventricular pathology.

Aortic stenosis often occurs in isolation and can result in reduced ventricular contractility, which commonly returns to normal once the outflow obstruction is removed, such as by TAVR. On the other hand, rendering the mitral valve competent again will require more work from the left ventricle (LV); this results in a well-described dip of the ventricular function and increased morbidity/mortality following intervention for MR. MR is also commonly associated with tricuspid regurgitation (TR) and atrial fibrillation, both of which impact morbidity and mortality independently. A tricuspid repair and anti-arrhythmia surgery are therefore routinely performed in patients undergoing mitral valve surgery. TMVR will not be a sufficient intervention in many patients that present with more than mild TR and/or atrial fibrillation. In fact, any TR is an exclusion criterion for all current clinical trials of TMVR. Transcatheter therapies for either TR or atrial fibrillation have not been able to reproduce the results seen with surgical intervention.

Paravalvular leak (PVL) has certainly remained as one of the Achilles' heels of THV implantation. In the case of TAVR, this has been greatly mitigated with new devices that have fabric coverage at the annular level and during procedures with post-implantation dilation of the prosthesis. The latter will prove much more problematic with the mitral valve, given the challenging combination of little anatomic support and proximity to the conduction system, circumflex coronary artery and aortic valve. Even if design of TMVR devices continues to improve, the slightest bit of PVL will be problematic in patients with long-term survival, given the high systolic pressure the prosthesis is exposed to. This is evidenced by the much higher mortality of mitral PVL compared with aortic PVL (12).

\section{TMVR device issues}

Multiple device-specific considerations distinguish TMVR from TAVR. The mitral valve annulus is significantly larger than the aortic annulus, and therefore will require larger valve mounted on larger delivery system. A substantial part 

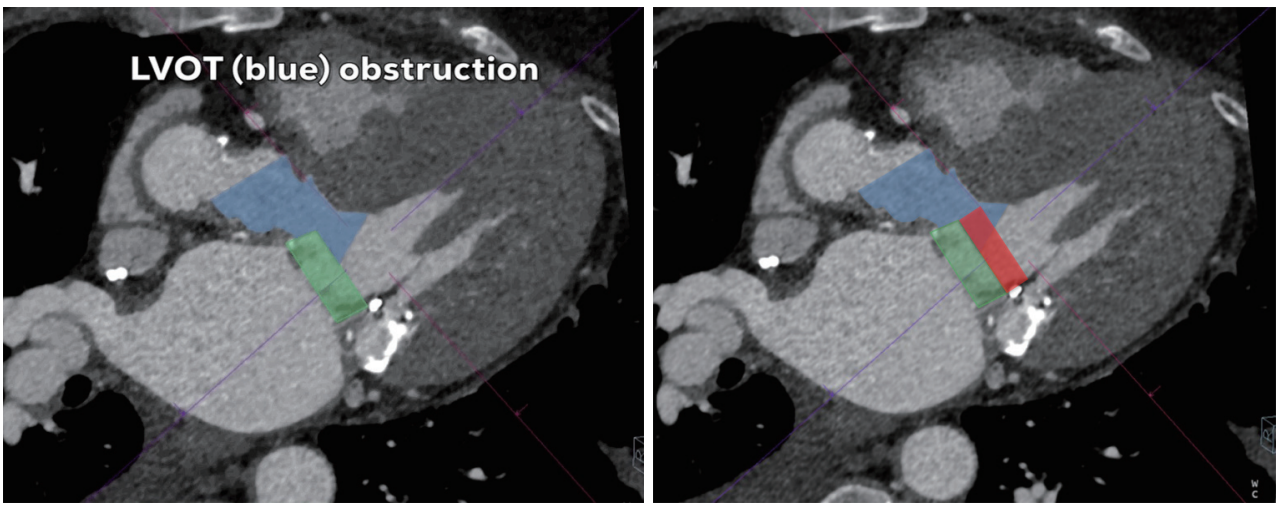

Figure 2 Progressive LVOT obstruction resulting from mitral valve prosthesis extending into LV cavity. Cardiac computed tomography showing a left ventricular (LV) long axis, mitral valve and LVOT. Blue color: LVOT and aortic root; green color: low profile mitral prosthesis; red color: high profile mitral prosthesis. LVOT, left ventricular outflow track.

of the success and rapid adoption seen with TAVR is related to the high success rate with extra-thoracic access used, such as transfemoral, transaxillary and transcarotid delivery. Several of the TMVR systems currently under investigation require transapical access and the larger system certainly makes any transseptal approach more challenging. The next challenge is the specific configuration of the mitral annulus and surrounding structures. While the aortic valve is situated in a complete fibrous ring, the mitral valve annulus is much more delicate and more or less supported along its circumference. Posteriorly, it is embedded in the junction of the left atrium and left ventricle, while the anterior portion essentially consists of the aorto-mitral curtain, a dynamic structure with limited rigidity. This poses multiple challenges for both securing a device, but also bares the risk of compression and distortion of other structures such as the aortic valve. If the mitral valve cannot be secured safely, the cyclic high systolic pressures it will be exposed to can easily lead to early or late device migration.

Furthermore, the D-shaped configuration of the mitral valve annulus poses a unique challenge. Firstly, appropriate sizing may prove difficult even with multimodality and fusion imaging, which have accomplished remarkable advances recently, and with the early lessons learned from TAVR. Early difficulties with TAVR and device sizing were related to the assumption that the aortic valve is round, while in fact it has an elliptical shape. Yet, the aortic annulus can be conformed to a relatively symmetrical round shape without much impact on surrounding structures, valve or cardiac function. This is not likely the case for the mitral valve. Secondly, the asymmetrical configuration of the mitral valve makes it difficult to achieve uniform radial force with any implant. This may lead to a higher incidence of device migration and PVL.

The proximity, and more importantly, the angle of the mitral valve annulus in relation to the LVOT further complicate TMVR. The device itself poses a risk of obstructing the LVOT. Additionally, the native anterior leaflet may cause obstruction being pushed anteriorly. This is akin to the obstruction of coronary ostia seen with TAVR, which is also gaining more recognition in the literature, and is particularly challenging if future access to the coronaries is needed. Several software packages and algorithms are currently available to obtain mitral annular sizing from cardiac computed tomography (CT) scans and to predict the extent to which a prosthesis will protrude into the LVOT (Figure 2). However, this is currently limited to making various assumptions and cannot reliably predict the extent of LVOT obstruction that will occur under dynamic conditions, potentially resulting in severe complications.

Finally, the requirements for approval of TMVR devices and the likely population for early trials is the same as for TAVR. This is problematic for various reasons. The landmark trials for TAVR were completed with two competing TAVR devices, meanwhile, there are currently at least 10 TMVR devices under investigation and another 20 devices are in the pipeline. Such competition will unquestionably dilute trial enrollment. More importantly, the frail, comorbid, high-risk or inoperable patient is a common scenario among individuals with degenerative aortic disease. On the other hand, this kind of demographic is rare in the larger pool of patients with mitral valve disease, 
that consists of mostly younger patients with degenerative MR. All this is reflected by the slow recruitment TMVR trials have seen across the board.

\section{Current status of TMVR trials}

In 2014, a conceptual milestone was reached when percutaneous implantation of a transcatheter valve in a surgical ring was first reported using the Edwards Sapien XT prosthesis (13). Several sporadic reports and anecdotal evidence suggest Sapien valves have been used occasionally to deliver a transcatheter aortic valve in the mitral position within a surgical valve, ring or in cases of severe MAC (14-16). The number of TMVRs with a dedicated mitral prosthesis and in a native mitral valve have remained relatively small to date with less than 150 human implants worldwide. The first such replacement was successfully completed in Denmark in 2012, using the CardiAQ prosthesis. Since then, the CardiAQ device has been used in several trials in an attempt to achieve CE mark approval in Europe; but most recently a trial was stopped by Edwards Lifesciences given concerns with the design of the valve. In the U.S., there are several centers that have participated in early TMVR trials. The Tendyne experience was recently reported as part of an international feasibility trial, in which 30 patients with a high surgical risk (STS 7.3\%) successfully had the transapically tethered TMVR valve implanted (17). Other valves that have entered clinical trials at various stages include the Neovasc Tiara, Medtronic Intrepid and Boston Scientific MValve devices, all competing with Tendyne to become the first commercially approved TMVR in Europe and then in the U.S. In addition, there is a large number of ongoing preclinical trials for several different devices (1).

The Intrepid study enrolled 50 patients who had severe, symptomatic mitral regurgitation and were at high or extreme risk for conventional mitral valve replacement (18). The device was successfully implanted in 48 patients. At 30 days, there were no disabling strokes or repeat interventions, while the mortality rate was 14 percent. In addition, 74 percent of patients had no mitral regurgitation at 30 days and the remaining 26 percent had mild disease.

The Tendyne study enrolled 30 patients with severe, asymptomatic mitral regurgitation who were deemed by the heart team to be poor candidates for surgery. The device was successfully implanted in 28 patients. At one year, five patients had died and three had been re-hospitalized for heart failure. None of the deaths were attributed to the procedure and no patients suffered a stroke. Of the other 22 patients, 21 had no mitral regurgitation at one year. At 30 days, transthoracic echocardiography showed mild central MR in one patient and no residual MR in the remaining 26 patients with valves in-situ. The left ventricular (LV) end-diastolic volume index decreased from mean $90.1 \mathrm{~mL} / \mathrm{m}^{2}$ at baseline to $72.1 \mathrm{~mL} / \mathrm{m}^{2}$. The Tendyne device is an apically tethered tri-leaflet porcine pericardial valve sewn onto a nitinol frame. It is specifically designed to address the complex mitral anatomy of functional, degenerative and mixed etiology mitral regurgitation. One of the major advantages of this device system is the fact that if the function of the prosthesis is not acceptable or LVOT obstruction occurs, it can be recaptured, repositioned, or fully retrieved, even after full deployment in the mitral annulus. The CardiAQ-Edwards Transcatheter Mitral Valve Replacement System (Edwards LifeSciences) is also being tested in an early feasibility study. In early 2017, Edwards paused enrollment of the trial to evaluate one of the valve's features. The company resumed the trial a few months later, announcing a decision to pursue a transseptal, rather than transapical, delivery approach. Finally, a small series has been reported on three patients suffering from functional mitral regurgitation with a severe reduction of $\mathrm{LV}$ function who received the Fortis TMVR device from Edwards under compassionate clinical use program because they were thought to be at very high risk for surgery (19).

The APOLLO trial using Medtronic's Intrepid valve is expected to enroll up to 1,200 patients with severe, symptomatic mitral regurgitation. A cohort of 650 patients who are candidates for surgery and not eligible for mitral repair will be randomized to receive the Intrepid or undergo surgery, while another cohort of up to 550 , deemed too high risk for surgery, will receive the Intrepid. All patients will be evaluated before and after the procedure, upon hospital discharge, and at 30 days, six months and on an annual basis for the next five years. All exclusion criteria (e.g., LV end-diastolic diameter $>70 \mathrm{~mm}$, severe mitral annular or leaflet calcification, left atrial or LV thrombus, prior mitral or aortic valve surgery, prior transcatheter mitral intervention, pulmonary artery systolic pressure $>70 \mathrm{mmHg}$, severe tricuspid regurgitation and severe right ventricular dysfunction) are quite common clinical, anatomic or hemodynamic characteristics of so-called inoperable or high-risk patients scheduled for mitral valve surgery, complicating the enrolment of patients in these trials. 


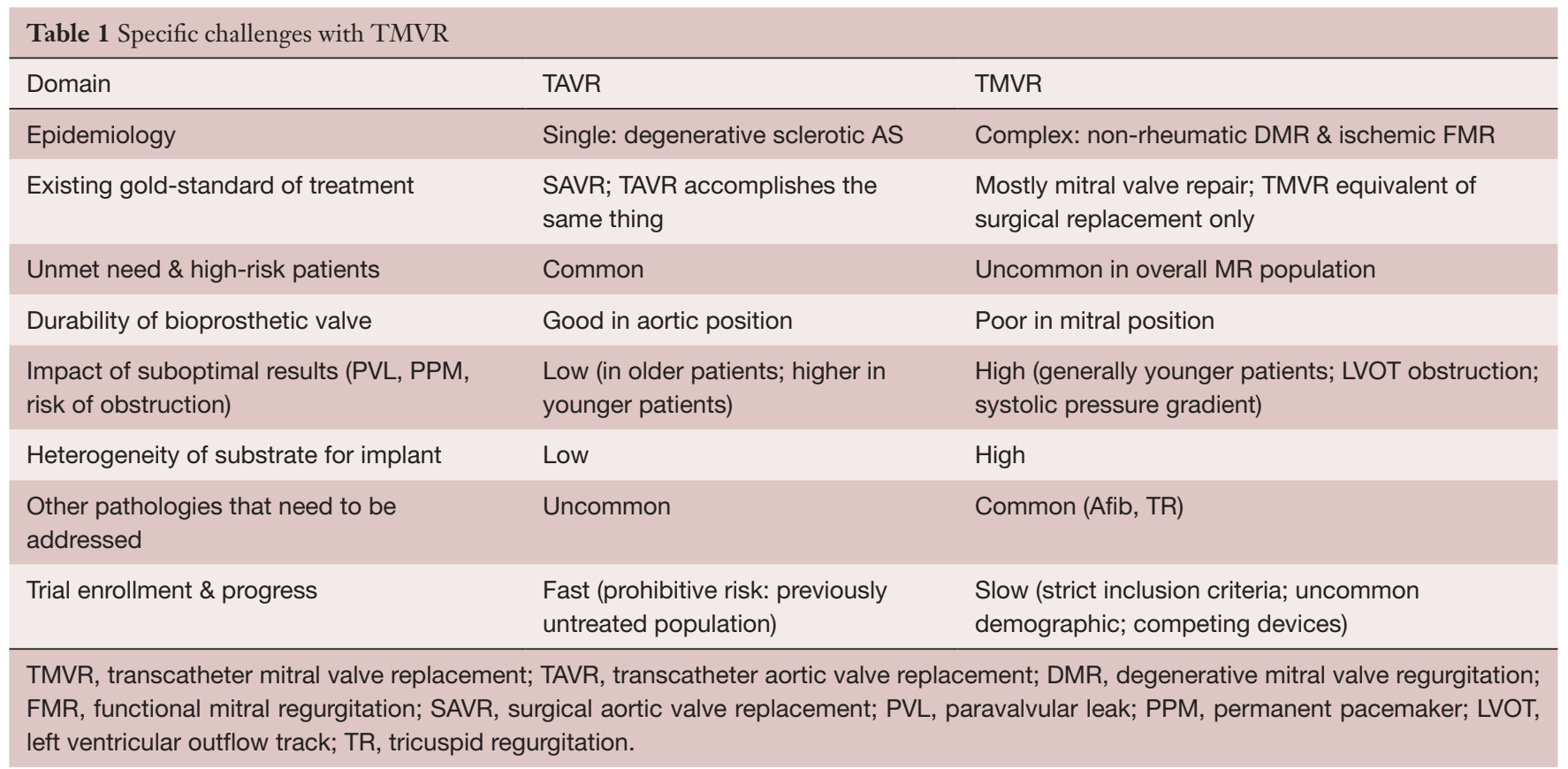

\section{Summary}

There are several important aspects that make the development and successful commercialization of TMVR devices challenging. These can be divided into several categories: the epidemiology and current treatment options for MR, the incidence and prevalence of different pathologies, anatomic and device challenges, as well as the trials currently underway for TMVR (Table 1). As less invasive methods will always be the preferred option, provided that outcomes are comparable with existing techniques and technology, TMVR will most certainly become a successful alternative treatment strategy in the long run. The current gold-standard for the management of patients with mitral valve disease consists of guidelinedirected medical management and open heart surgery. Contemporary results of mitral valve surgery demonstrate a very high repair rate in non-rheumatic degenerative mitral valve disease with excellent long-term outcomes and low mortality (1.1\%) (20). Review of the STS database and cases performed at the US Veterans Administration Health System also clearly show the increased mortality that is associated with mitral valve replacement in patients with degenerative MR (21). For ischemic functional MR, recent randomized clinical trials have shown comparable results between mitral valve repair and replacement in terms of mortality and somewhat favorable results for replacement with regards to residual MR. As TMVR tries to make its way into clinical practice, these surgical standards will provide the benchmark. Simultaneously, the devices will be expected to be safe, reliable and provide durable results consistent with the current surgical experience of mitral valve replacement using a bioprosthetic valve. In order to offer a truly minimally-invasive alternative to surgery, delivery will also have to be delivered by a trans-septal approach. Efforts to this extent are well underway and TMVR can be expected to be commercially available for select patient groups within the next few years.

\section{Acknowledgements}

Dr. Wyler von Ballmoos received grant support from the Thoracic Surgery Foundation for Research and Education via the Michael J. Davidson Fellowship Award.

\section{Footnote}

Conflicts of Interest: The authors have no conflicts of interest to declare.

\section{References}

1. Badhwar V, Thourani VH, Ailawadi G, et al. Transcatheter mitral valve therapy: The event horizon. J Thorac 
Cardiovasc Surg 2016;152:330-6.

2. Nkomo VT, Gardin JM, Skelton TN, et al. Burden of valvular heart diseases: a population-based study. Lancet 2006;368:1005-11.

3. Bursi F, Enriquez-Sarano M, Nkomo VT, et al. Heart failure and death after myocardial infarction in the community: the emerging role of mitral regurgitation. Circulation 2005;111:295-301.

4. Chatterjee S, Rankin JS, Gammie JS, et al. Isolated mitral valve surgery risk in 77,836 patients from the Society of Thoracic Surgeons database. Ann Thorac Surg 2013;96:1587-94; discussion 1594-5.

5. Brown JM, O'Brien SM, Wu C, et al. Isolated aortic valve replacement in North America comprising 108,687 patients in 10 years: changes in risks, valve types, and outcomes in the Society of Thoracic Surgeons National Database. J Thorac Cardiovasc Surg 2009;137:82-90.

6. Thourani VH, Suri RM, Gunter RL, et al. Contemporary real-world outcomes of surgical aortic valve replacement in 141,905 low-risk, intermediate-risk, and high-risk patients. Ann Thorac Surg 2015;99:55-61.

7. Lim ZY, Boix R, Prendergast B, et al. First reported case of transcatheter mitral valve implantation in mitral annular calcification with a fully repositionable and self-expanding valve. Circ Cardiovasc Interv 2015;8:e003031.

8. Nishimura RA, Otto CM, Bonow RO, et al. 2017 AHA/ ACC Focused Update of the 2014 AHA/ACC Guideline for the Management of Patients With Valvular Heart Disease: A Report of the American College of Cardiology/ American Heart Association Task Force on Clinical Practice Guidelines. J Am Coll Cardiol 2017;70:252-89.

9. Castillo JG, Anyanwu AC, Fuster V, et al. A near 100\% repair rate for mitral valve prolapse is achievable in a reference center: implications for future guidelines. J Thorac Cardiovasc Surg 2012;144:308-12.

10. Goldstein D, Moskowitz AJ, Gelijns AC, et al. Two-Year Outcomes of Surgical Treatment of Severe Ischemic Mitral Regurgitation. N Engl J Med 2016;374:344-53.

11. Michler RE, Smith PK, Parides MK, et al. Two-Year Outcomes of Surgical Treatment of Moderate Ischemic

Cite this article as: Wyler von Ballmoos MC, Kalra A, Reardon MJ. Complexities of transcatheter mitral valve replacement (TMVR) and why it is not transcatheter aortic valve replacement (TAVR). Ann Cardiothorac Surg 2018;7(6):724-730. doi: 10.21037/acs.2018.10.06
Mitral Regurgitation. N Engl J Med 2016;374:1932-41.

12. Cho IJ, Moon J, Shim CY, et al. Different clinical outcome of paravalvular leakage after aortic or mitral valve replacement. Am J Cardiol 2011;107:280-4.

13. Maisano F, Reser D, Pavicevic J, et al. Successful firstin-man Melody transcatheter valve implant in a dehisced mitral annuloplasty ring transapical valve-in-ring implant. EuroIntervention 2014;10:961-7.

14. Fassa AA, Himbert D, Brochet E, et al. Transseptal transcatheter mitral valve implantation for severely calcified mitral stenosis. JACC Cardiovasc Interv 2014;7:696-7.

15. Sinning JM, Mellert F, Schiller W, et al. Transcatheter mitral valve replacement using a balloon-expandable prosthesis in a patient with calcified native mitral valve stenosis. Eur Heart J 2013;34:2609.

16. Praz F, Windecker S, Huber C, et al. Expanding Indications of Transcatheter Heart Valve Interventions. JACC Cardiovasc Interv 2015;8:1777-96.

17. Muller DW, Farivar RS, Jansz P, et al. Transcatheter Mitral Valve Replacement for Patients With Symptomatic Mitral Regurgitation: A Global Feasibility Trial. J Am Coll Cardiol 2017;69:381-91.

18. Bapat V, Rajagopal V, Meduri C, et al. Early Experience With New Transcatheter Mitral Valve Replacement. J Am Coll Cardiol 2018;71:12-21.

19. Abdul-Jawad Altisent O, Dumont E, Dagenais F, et al. Initial Experience of Transcatheter Mitral Valve Replacement With a Novel Transcatheter Mitral Valve: Procedural and 6-Month Follow-Up Results. J Am Coll Cardiol 2015;66:1011-9.

20. Gammie JS, Chikwe J, Badhwar V, et al. Isolated Mitral Valve Surgery: The Society of Thoracic Surgeons Adult Cardiac Surgery Database Analysis. Ann Thorac Surg 2018;106:716-27.

21. Bakaeen FG, Shroyer AL, Zenati MA, et al. Mitral valve surgery in the US Veterans Administration health system: 10-year outcomes and trends. J Thorac Cardiovasc Surg 2018;155:105-17.e5. 